\title{
Approximate Assessment of the Ultimate Longitudinal Strength of the Hull Girder
}

\author{
J. M. Gordo, ${ }^{1}$ C. Guedes Soares, ${ }^{1}$ and D. Faulkner ${ }^{2}$
}

\begin{abstract}
A method is presented to estimate the ultimate moment based on a simplified approach to represent the behavior of stiffened plate columns. The assessment of the strength of a very large crude carrier is performed and compared with the moment at failure in hogging estimated by other methods applied to the same case. The proposed method allows the prediction of the degradation of the strength due to corrosion and residual stresses. It also allows the evaluation of the strength of the hull at several heeling conditions. Finally, an analysis of the efficiency of the high tensile steel is carried out.
\end{abstract}

\section{Introduction}

THE earliest attempts to incorporatc the effects of plate buckling on ship strength assessment were made by Caldwell (1965), who used a simplified procedure where the ultimate moment of a midship cross section in the sagging condition was calculated, introducing the concept of a structural instability strength reduction factor for compressed panels. Faulkner (1975) further developed the concept by suggesting a design method to calculate this reduction factor.

Smith et al $(1977,1986)$ and Dow et al $(1981)$ developed an incremental curvature procedure which allows the derivation of a moment-curvature relationship for a complete hull. It is a hybrid method based mainly on a finite-element formulation but where the plate element strength is obtained from a set of empirical curves.

Billingsley (1980) used an engineering approach which considered a very simplified model for each individual beamcolumn element. The strength of the hull girder was obtained from the summation of the individual contributions of each element.

While the early attempts were based on the collapse strength of an individual plate, more recent ones have considered the sequence of collapsing plates. Adamchak (1984) has developed a simplified method, together with a computer program which implements it, where the ultimate strength of each panel includes a flexural-torsional buckling formulation. Curves of moment-curvature are built from a set of discrete points corresponding to the buckling of each panel.

Lin (1985) described a similar method but he considered a different approach to assess the plate strength and used a dynamic relaxation method for the stiffened panel strength. Several comparisons were made with experimental results, from which two simplified expressions for ultimate moment prediction were presented.

Rutherford \& Caldwell (1990) recently presented a comparison between the ultimate bending moment experienced by a very large crude carrier (VLCC) and the results of retrospective strength calculations in which a simplified approach to stiffened plates collapse was used, but without con-

${ }^{1}$ Unit of Marine Technology and Engineering, Instituto Superior Técnico, Universidade Técnica de Lisboa, Lisbon, Portugal.

${ }^{2}$ Department of Naval Architecture and Ocean Engineering, University of Glasgow, Glasgow, Scotland.

Revised manuscript received at SNAME headquarters September 15,1995 sidering the post-buckling behavior. Also, the importance of lateral pressure, initial deformations, and corrosion rates was investigated. The validity of the model and method was confirmed by a nonlinear finite-element program.

In addition to these simplified methods, a fully nonlinear finite-element analysis has been performed, for example, by Kutt et al (1985), but this was shown to be a very timeconsuming task both in modeling the structure and in computing time.

The present work was developed in order to provide a computationally inexpensive procedure to assess the ultimate longitudinal strength with adequate accuracy. The method was checked by comparing its predictions with those of Kutherford \& Caldwell for the VLCC Energy Concentration. After being checked, the method was used to study the effects of other phenomena such as residual stresses, corrosion, and combined bending in order to illustrate its capability.

\section{The method}

The present method follows the general approach presented by Billingsley (1980) and by Rutherford \& Caldwell (1990) but, with respect to plate strength and beam-column behavior, the models reported in Gordo \& Guedes Soares (1993) were used. These models are based on the Faulkner formulations to describe the ultimate strength of plate elements and of the stiffened plate columns. Both models were extended to predict the behavior of plates and stiffened plates within the usual ranges of strains.

Broadly speaking, the moment-curvature relationship is determined by imposing a set of curvatures on the hull's girder. For each curvature the state of the average strain of each beam-column element is determined. Entering with these values in the load-shortening curves, the load sustained by each element may be calculated. The bending moment sustained by the cross section is obtained from the summation of the moments of the forces in the individual elements. The derived set of values defines the desired moment-curvature relation.

Some problems arise, however, in the implementation of the present method; for example the sequence and spacing of imposed curvatures strongly influence the convergence of the method due to the shift of the neutral axis. Also, modeling of the ship's section and determining the position of the neutral axis itself are important issues.

The basic assumptions of the method are:

- The elements into which the cross section is subdivided are considered to act and behave independently. 
- Plane sections are assumed to remain plane when curvature is increasing; this condition is necessary to estimate the strain level of the elements, but its validity is doubtful when shear is present in plate elements.

- Overall grillage collapse is avoided by sufficiently strong transverse frames.

As a first step it is necessary to estimate the position of the neutral axis through an elastic analysis because, when the curvature is small, the section behaves elastically. The elastic neutral axis passes through a point given by

$$
\left\{\begin{array}{l}
G_{x}=0 \text { due to symmetry } \\
G_{y}=\frac{\sum y_{i} A_{i}}{\Sigma A_{i}}
\end{array}\right.
$$

where the origin is centered on the intersection of the baseline and centerline, Fig. 1.

The most general case corresponds to that in which the ship is subjected to curvature in the $x$ - and $y$-directions, respectively denoted as $C_{x}$ and $C_{y^{*}}$. The global curvature $C$ is related to these two components by

$$
C=\sqrt{C_{x}^{2}+C_{y}^{2}}
$$

or by

$$
\left\{\begin{array}{l}
C_{x}=C \cdot \cos \theta \\
C_{y}=C \cdot \sin \theta
\end{array}\right.
$$

adopting the right-hand rule, where $\theta$ is the angle between the neutral axis and the $x$-axis. The strain at the centroid of any element $\epsilon_{i}$ is consequently

$$
\epsilon_{i}=y_{g i} \cdot C_{x}-x_{g i} \cdot C_{y}
$$

or from (3)

$$
\epsilon_{i}=C \cdot\left(y_{g i} \cdot \cos \theta-x_{g i} \cdot \sin \theta\right)
$$

where $\left(x_{g i}, y_{g i}\right)$ is the vector from the centroid of the section to the centroid of the element $i$ (stiffener and associated effective plate). The relation between these local coordinates and the global coordinates is

$$
\begin{aligned}
& x_{g i}=x_{i}-G_{x} \\
& y_{g i}=y_{i}-G_{y}
\end{aligned}
$$

Equations (4) and (5) are still valid if one uses any point belonging to the neutral axis instead of the point of the intersection of the axes resulting from horizontal and vertical equilibrium of the longitudinal forces due to the bending moment.

Once the strain state of each element is achieved, the corresponding average stresses may be calculated according to expressions in Gordo \& Guedes Soares (1993) and Gordo

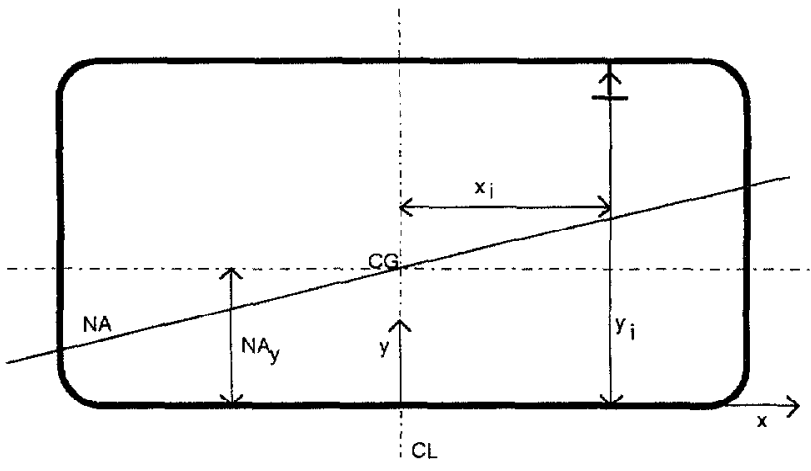

Fig. 1 Combined bending of hull girder

(1993), and consequently the components of the bending moment at a curvature $C$ are

$$
\left\{\begin{array}{l}
M_{x}=\Sigma y_{g i} \cdot \Phi\left(\bar{\epsilon}_{i}\right) \cdot \sigma_{o} A_{i} \\
M_{y}=\Sigma x_{g i} \cdot \Phi\left(\bar{\epsilon}_{i}\right) \cdot \sigma_{o} A_{i}
\end{array}\right.
$$

where $x_{g i}$ and $y_{g i}$ are the distances from the element $i$ to a local axis of a reference datum located in the precise position of the instantaneous "center of gravity" (CG) and $\bar{\epsilon}_{i}$ is the nondimensional strain of the element $i$ at the instantaneous curvature.

The modulus of the total amount is

$$
M=\sqrt{M_{x}^{2}+M_{y}^{2}}
$$

which is the bending moment on the cross section if the instantaneous CG is placed in the correct location. Along the step-by-step process, however, this location shifts and so it is necessary to calculate the shift between the two imposed curvatures. Rutherford \& Caldwell (1990) suggested that the shift could be taken equal to

$$
\Delta G=\frac{\Sigma\left(A_{i} \cdot \sigma_{i}\right)}{C \cdot \Sigma\left(A_{i} \cdot E_{i}\right)}
$$

but it was felt that this expression underestimated the shift and may cause problems in convergence.

For this reason a trial-and-error process was implemented having, as stopping criteria, one of two conditions: the total net load in the section, $N L$, or the error in the shift estimation $\Delta G$ should be less than or equal to sufficiently low values. Equations (11) and (12) represent analytically these two conditions, where $\xi$ was taken equal to $10^{-6}$.

$$
\begin{aligned}
& N L=\Sigma\left(\sigma_{i} \cdot A_{i}\right) \leqslant \xi \cdot \sigma_{o} \cdot \Sigma\left(A_{i}\right) \\
& \Delta G=k_{E} \cdot \frac{N L}{C \cdot E \cdot \Sigma A_{i}} \leqslant 0.0001
\end{aligned}
$$

\section{Nomenclature}

\footnotetext{
$A_{i}=$ sectional area of element $i$

$C=$ curvature

$C_{x}=$ curvature over $x$-axis

$C_{y}=$ curvature over $y$-axis

$E=$ Young's modulus of material

$E_{i}=$ tangent modulus of element $i$

$G_{x}=$ instantaneous abcissa of the centroid

$G_{y}=$ instantaneous ordinate of the centroid

$l=$ length between frames

$M=$ magnitude of bending moment

$M_{x}=$ component of moment about $x$-axis

$M_{y}=$ component of moment about $y$-axis

$N L=$ net load of section

$r=$ radii of gyration of cross section of element

$x_{g i}=$ horizontal distance of centroid of element $i$ to instantaneous center of gravity (CG)

$x_{i}=$ distance of centroid of element $i$ to centerline

$y_{g i}=$ vertical distance of centroid of element $i$ to instantaneous $\mathrm{CG}$

$y_{i}=$ distance of centroid of element $i$ to baseline
}

$\epsilon_{i}=$ strain of element $i$

$\epsilon_{0}=$ yield strain

$\eta=$ tensile width due to residual stresses normalized by thickness

$\lambda=$ column slenderness $\left(=l / r \sqrt{\left.\sigma_{0} / E\right)}\right.$

$\sigma_{i}=$ average stress in element $i$

$\bar{\kappa}_{r}=$ normalized residual stress

$\theta=$ angle between vector of curvature and $x$-axis

$\Phi\left(\epsilon_{i}\right)=$ average stress of element $i$ at a strain of $\epsilon_{i}$ normalized by yield stress

$\Delta G=$ shift of neutral axis 
The factor $k_{E}$ is a function of the curvature and yield strain introduced to permit a better convergence of the method, and it is a result of the variation in global tangent modulus of the section with curvature.

\section{Strength of stiffened plate columns}

The compression strength of the stiffened plate column at a certain strain, $\Phi\left(\bar{\epsilon}_{i}\right)$ of equation $(8)$, is given by the minimum of two independent functions, one representing the strength due to flexural buckling $\Phi_{b}\left(\bar{\epsilon}_{i}\right)$ and the other due to tripping of the stiffener $\Phi_{t}\left(\bar{\epsilon}_{i}\right)$

$$
\Phi_{b}\left(\bar{\epsilon}_{i}\right)=\Phi_{j o}\left(\bar{\epsilon}_{i}\right) \cdot \frac{A_{S}+\Phi_{w}\left(\bar{\epsilon}_{i}\right) \cdot b t}{A_{S}+b t}
$$

where $\Phi_{j o}\left(\bar{\epsilon}_{i}\right)$ is based on the Johnson-Ostenfeld formulation accounting for inelastic effects on the column's buckling as defined in Gordo \& Guedes Soares (1993). The second term computes the loss of efficiency of the plate due to compression loading. In fact, $\Phi_{w}\left(\bar{\epsilon}_{i}\right)$ is the effective width of the plate of the element at a strain $\bar{\epsilon}_{i}$, based on the Faulkner (1975) approach to the plate strength, and given by

$$
\Phi_{w}\left(\bar{\epsilon}_{i}\right)=\Phi_{e}\left(\bar{\epsilon}_{i}\right) \cdot\left(\frac{2}{\beta}-\frac{1}{\beta^{2}}\right)
$$

where $\beta$ is the slenderness of the plate as a function of the instantaneous strain so that $\beta=(b / t) \sqrt{\epsilon_{i}}$, and $\Phi_{e}\left(\bar{\epsilon}_{i}\right)=$ $\max \left(-1, \min \left(1, \bar{\epsilon}_{i}\right)\right)$ is the elastic-perfectly plastic behavior of the material at the same strain. The second term of the second member of equation (14) resumes to 1 for $\beta<1$, which is the case of the plate under tension and this means that the plate is considered to be fully effective.

The tripping strength, $\Phi_{t}\left(\bar{\epsilon}_{i}\right)$, is given by:

$$
\Phi_{t}\left(\bar{\epsilon}_{i}\right)=\Phi_{t \min } \cdot \frac{\epsilon_{t}}{\epsilon_{i}} \cdot \frac{A_{s}+\Phi_{w}\left(\bar{\epsilon}_{i}\right) \cdot b t}{A_{s}+b t}
$$

which accounts for the minimum elastic tripping stress, $\Phi_{t \min }$ (Adamchack 1979, Faulkner 1974, 1987), for the load shedding pattern and the strain of maximum load (second term) and for the loss of efficiency of the plate (third term). The strain of maximum load due to tripping is defined by $\epsilon_{t}$ $=\Phi_{i \text { min }} \cdot \epsilon_{o}$.

Figure 2 illustrates the unstiffened and stiffened plate be- havior as function of the normalized strain for a bottom element of Energy Concentration.

\section{Modeling of the ship's cross section}

As hull strength assessment is based on the strength of stiffened panels, the modeling of the ship's cross section consists of discretizing the hull into stiffened plate elements which are representative of panel behavior. The design philosophy of the shipbuilding industry is oriented mainly to longitudinal stiffened hulls. In these hulls it is common practice currently to have panels with similar and repetitive properties such as space between stiffeners, thicknesses, and stiffener geometry.

As the behavior of these panels may be represented as the behavior of $n$ equally stiffened plate elements, then the hull section will be divided into small elements representing a plate between stiffeners and the corresponding stiffener. Apart from the validity of this modeling concerning panel behavior (Gordo \& Guedes Soares 1993), some other points of the modeling involve approximations, especially related to the

- validity of the element's stress state as derived from the strain state at the centroid of the element,

- modeling of side girders when web stiffeners are not present,

- modeling of corners, and

- modeling of large and rcinforced flanges of the primary longitudinal girder system and the validity of this subdivision on the overall behavior of main girders, especially relating to sideways flexural behavior.

Relating to the first point, Fig. 3 shows the strain distribution in a reinforced element. The stress state of the components of the element (plate and stiffener) is computed considering the strain at the centroid $\epsilon_{i}$. However, the real plate strain is higher than that strain and is approximately equal to $\epsilon_{\max }$. This error is only relevant when the element belongs to horizontal panels, i.e., deck and bottom, but because in these two structures the distance of the elements from the neutral axis is very high compared with the dimensions of the elements, it results in a ratio between these two strains close to one, which makes the approximation acceptable and allows the simplification of the procedures and iterations.

For the contribution of the side girders without longitudi-

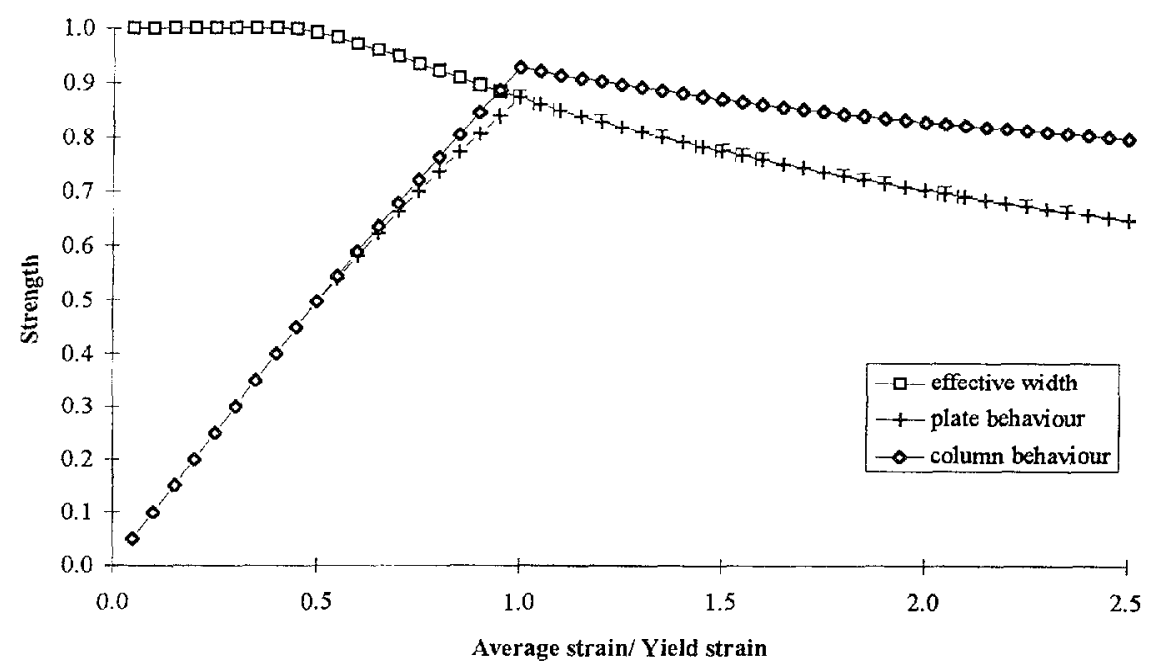

Fig. 2 Average stress-average strain curves of an unstiffened plate with a nominal slenderness of $\beta=$ 1.56 and of corrcsponding stiffened plate with a column slenderness of $\lambda=\operatorname{li} r \sqrt{\sigma_{d} J}=0.61$, representing bottom panel of Energy Concentration. Tripping is not predicted for this panel 


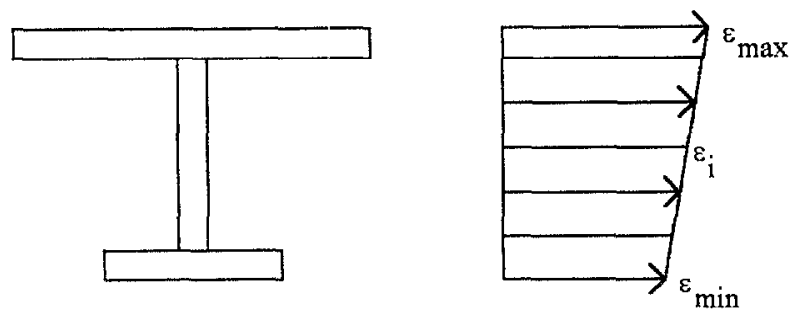

Fig. 3 Strain state of a stiffened plate element

nal stiffeners, two solutions are possible: first, to neglect their contribution due to the low level of the bending moment experienced as a consequence of the low strength and the proximity of the neutral axis, or second, to calculate approximately the strength using the formula for transversely loaded plates (Guedes Soares \& Gordo 1996).

In this work the latter solution is adopted and the girders are modeled considering three main components: the flange with a stiffener (which is not more than the edge of the girder's web), another stiffened plate on the other side of the girder consisting of side plating and part of the web, and a central plate region, Fig. 4. The extremes are modeled as beam columns in order to compute their higher rigidity compared with that of plate elements alone; the breadth of the stiffeners (part of the web) must be carefully chosen by the designer in such a way that they behave as "hard" elements (low column slenderness).

Relative to the contribution of the corners, normally three situations are considered: first, behaving like hard corners, i.e., considering that their strength is one $(\phi=1)$ in the plastic domain; second, not taking into account any contribution of the corners on the hull's strength; and third, modeling corners as usual beam columns. Any of these solutions is formally unsatisfactory because they do not include the influence of the buckled state of adjacent panels on the behavior of the corner.

\section{Analysis of Energy Concentration}

In order to test the method and the computer program developed, the hull of the VLCC Energy Concentration was chosen because several studies that have been completed for this ship permit good comparisons to be made.

This VLCC broke her back in July 1980 during a discharge of oil at Rotterdam. As the ship was discharging in still water, and the load conditions were fairly well known, the determination of the ultimate bending moment of the ship could be computed with sufficient accuracy.

\section{The ship}

The ship was ten years old and had been constructed by Kawasaki Heavy Industries, in March 1970, in Japan, according to the current design practice at the time for VLCC's and had the principal dimensions given in Table 1.

Figure 5 shows the profile and plan of the ship and the general arrangement of cargo, ballast and slop tanks, as well

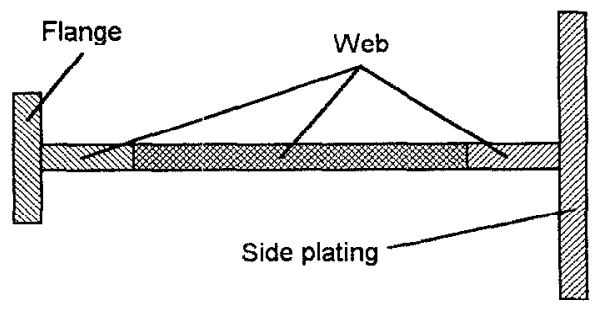

Fig. 4 Modeling of unstiffened side girders
Table 1 Particulars of VLCC Energy Concentration

\begin{tabular}{lc}
\hline Length, overall & $326.75 \mathrm{~m}$ \\
Length, between perpendiculars & $313.00 \mathrm{~m}$ \\
Molded breadth & $48.19 \mathrm{~m}$ \\
Molded depth & $25.20 \mathrm{~m}$ \\
Draft, summer & $19.60 \mathrm{~m}$ \\
Deadweight & 216269 tons \\
\hline
\end{tabular}

as the location of longitudinal and transverse bulkheads. The ship was initially classified by Det norske Veritas and had changed to Bureau Veritas in 1977. The last survey report found all cargo and ballast tanks in good condition, with the exception of ballast tanks No. 3, where a greater degree of corrosion was found. The location of these tanks is coincident with the location of the failure.

At the beginning of her last voyage, the ship was heavily trimmed by the stern $(1.47 \mathrm{~m})$ and showed a hog deflection of $12 \mathrm{~cm}$. Further calculations performed by Rutherford \& Caldwell (1990), Fig. 6, concluded that the failure hogging moment should be about $17940 \mathrm{MN}$ - m. It is also possible to detect a region on the aft side of frame 76 where very high levels of moment and shear coexisted due to the fact that the central tanks were empty.

\section{Modeling of the ship}

In order to perform the analysis of the ultimate carrying capacity of Energy Concentration and to determine the moment-curvature relationship, it is necessary to model the cross section in stiffened elements as was proposed in Section 4. Figure 7 shows the solution adopted for half of the section, which has 242 reinforced elements and 2 plate elements. Figures 7 and 8 together with Table 2 give the relevant information about the dimensions and material properties of the section and elements. Note that the ship uses two different kinds of material in three regions: the deck and bottom are of high-strength steel (HTS, $\sigma_{o}=315 \mathrm{MPa}$ ) and the main parts of side shell, longitudinals, and bulkheads are constructed of mild steel (MS, $\sigma_{o}=235 \mathrm{MPa}$ ). The frame spacing in the zone of interest is $5.10 \mathrm{~m}$ and the spacing between longitudinals varies between $925 \mathrm{~mm}$ on the side and bulkheads and 1000 mm on the deck and bottom, Fig. 9 .

\section{Moment-curvature curve}

The program developed is able to predict the moment-curvature relationship for several conditions of heeling, levels of corrosion, residual stresses and distortions, and also to deal with the effect of load shedding after the buckling of the panels on the ultimate bending moment of the section.

In this subsection the results obtained for the initial cross section of the Energy Concentration are compared with those obtained by the Rutherford \& Caldwell (1990) analysis and the calculated moment at the time of failure, Table 3 . The results obtained (columns 2 and 3 ) show that the predictions are $7 \%$ above the estimated failure moment of the ship for the "as built" condition. However, it has been noted that the ship at the time of failure was ten years old and showed some degree of corrosion in the region of failure.

Also, the results of the applied method do not account for any level of residual stresses, which seems to be in accordance with reality due to their shakeout during operation time. In relation to distortions, Faulkner's equation for plates incorporates itself the effect of average levels of plate distortions; thus this effect is accounted for. However, the results in Table 3 do not incorporate explicitly the stiffener out-ofplane distortions and an increase in their magnitude reduces the ultimate moment.

The last four columns in Table 3 compare the results taking into account a standard level of corrosion of $1 \mathrm{~mm}$. The 

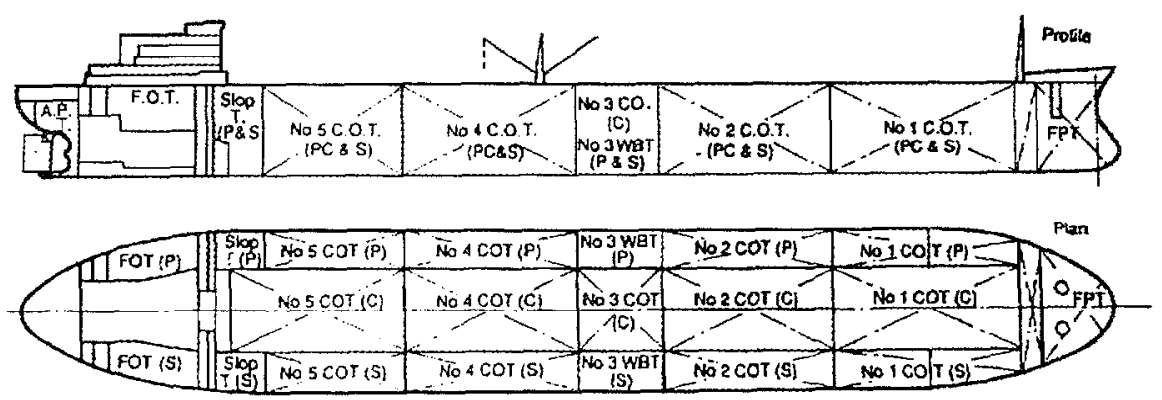

Fig. 5 Profile of VLCC Energy Concentration

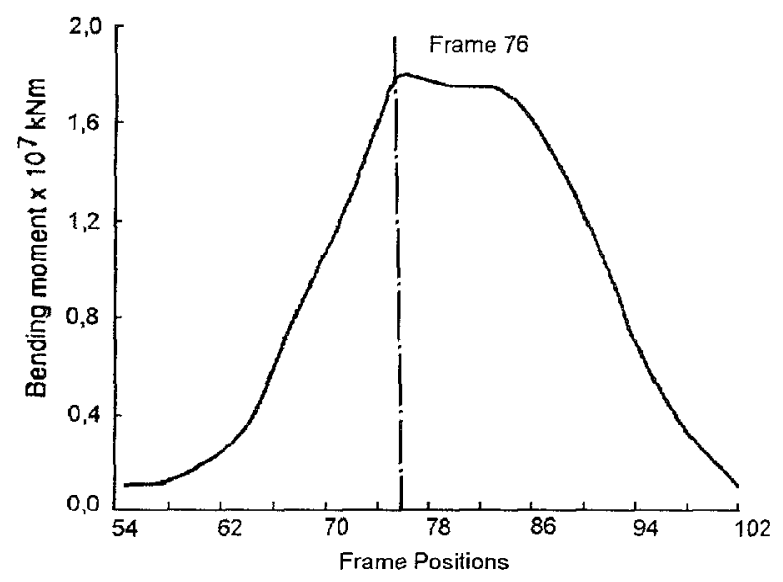

Fig. 6 Bending moment distribution at failure time

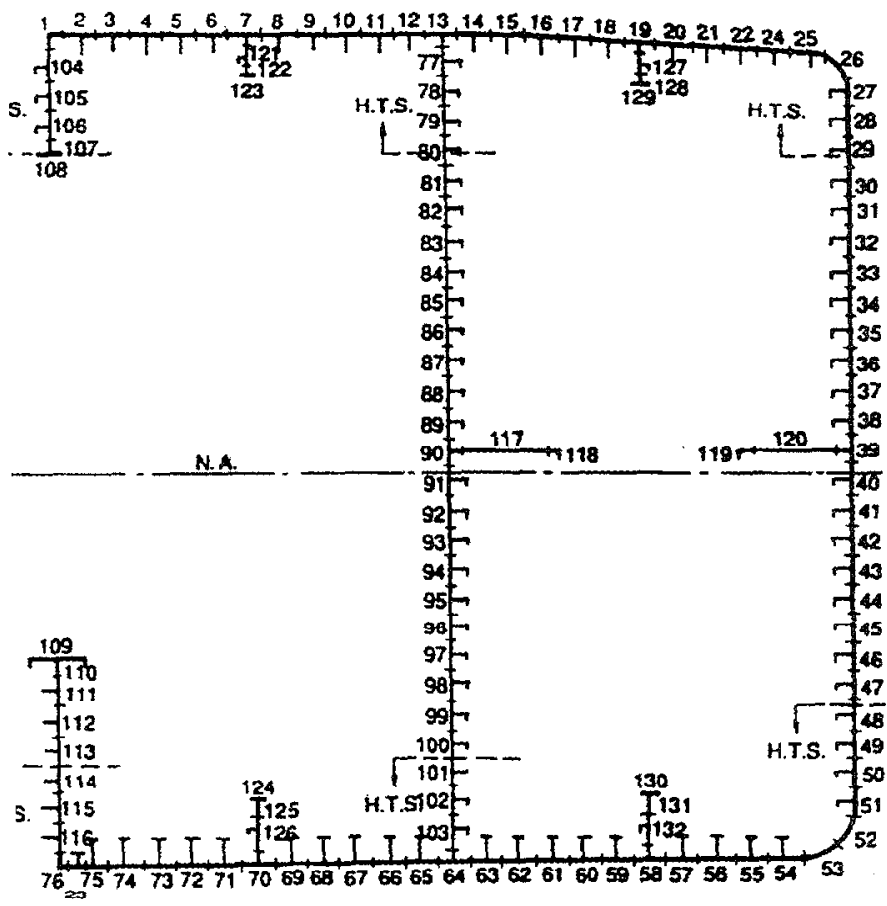

Fig. 7 Modeling of failure cross section

results are all within a band of $5 \%$, which demonstrates the validity of the simplified method proposed here. Moreover, the predicted value for the ultimate moment in hogging is technically equal to the estimated moment at failure.

Referring to the moment-curvature curve itself, Fig. 10 shows that the maximum moment occurs at an approximate curvature of $1.21 \times 10^{-7} \mathrm{~mm}^{-1}$, and after that point a smooth decrease of the bending moment sustained is present.

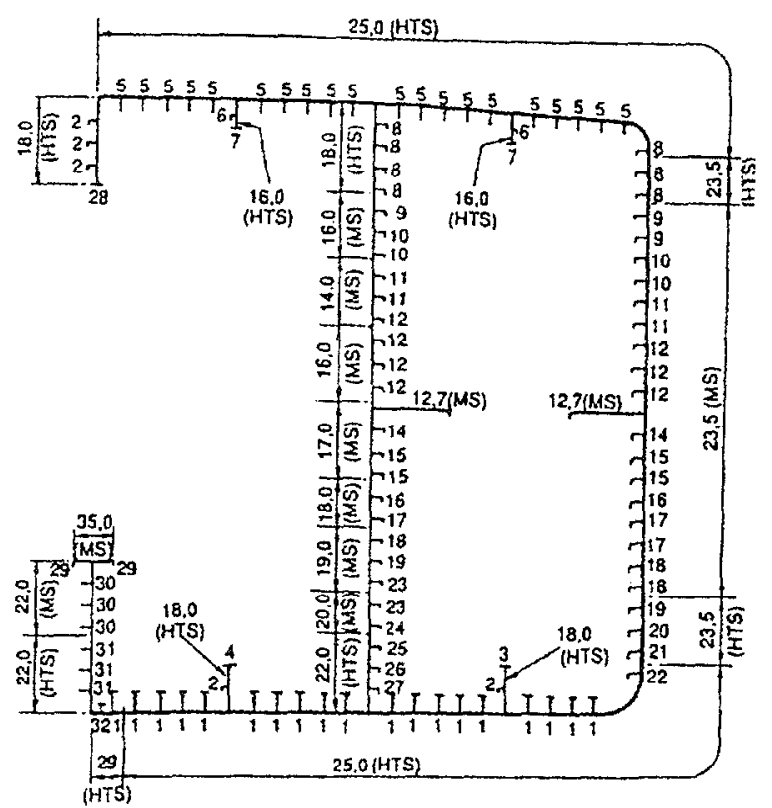

Fig. 8 Scantlings of plating and longitudinals for Energy Concentration

The curves of the position of the neutral axis show three main regions:

1. An elastic region from zero to a curvature of about half the ultimate curvature.

2. A region of progressive loss of plate effectiveness due to compression and corresponding to a smooth change in the neutral axis position, which extends until the ultimate curvature; this region includes a very narrow band near ultimate curvature where the effect of the yield of the panels in tension (deck panels in hogging) equilibrates the increasing loss of effective width of the plating in compression.

3. A load-shedding region, in which the position of the neutral axis is moving rapidly due to the collapse of the most compressed panels.

Table 3 also compiles the ultimate moment results for sagging. It is important to note that the ultimate bending moment in sagging is about $20 \%$ lower than the yield bending moment for all methods, which demonstrates the weakness of using the elastic theory as a basis for the rules of classification societies. The yield bending moment is defined as the moment corresponding to the first yield, normally in the deck, when an elastic analysis of the stresses in the section is performed.

The results from the method proposed are higher than those calculated by Lloyd's Program No. 20202 (Rutherford \& Caldwell results) in sagging ( $5 \%$ ) while they give the "same" results in hogging. This discrepancy may be a result of applying different formulations for tripping behavior of stiffeners and also from the assumed load shedding pattern after 
Table 2 Stiffener scantlings and material

\begin{tabular}{|c|c|c|c|c|c|c|c|}
\hline Stf. $n$. & Web & Flange & Steel & Stf. & Web & Flange & Steel \\
\hline 1 & $797 \times 15$ & $200 \times 33$ & HTS & 17 & $747 \times 12.7$ & $180 \times 25$ & MS \\
\hline 2 & $297 \times 11.5$ & $100 \times 16$ & HTS & 18 & $797 \times 14$ & $180 \times 25$ & MS \\
\hline 3 & $370 \times 16$ & & HTS & 19 & $847 \times 14$ & $180 \times 25$ & MS \\
\hline 4 & $425 \times 25$ & & HTS & 20 & $847 \times 14$ & $180 \times 32$ & MS \\
\hline 5 & $480 \times 32$ & & HTS & 21 & $847 \times 15$ & $180 \times 25$ & HTS \\
\hline 6 & $297 \times 11.5$ & $100 \times 16$ & HTS & 22 & $847 \times 15$ & $180 \times 32$ & HTS \\
\hline 7 & $370 \times 16$ & & HTS & 23 & $897 \times 15$ & $200 \times 25$ & MS \\
\hline 8 & $447 \times 11.5$ & $125 \times 22$ & HTS & 24 & $945 \times 16$ & $200 \times 25$ & MS \\
\hline 9 & $549 \times 11.5$ & $125 \times 22$ & MS & 25 & $897 \times 15$ & $200 \times 25$ & HTS \\
\hline 10 & $597 \times 11.5$ & $125 \times 22$ & MS & 26 & $797 \times 15$ & $180 \times 25$ & HTS \\
\hline 11 & $597 \times 11.5$ & $125 \times 25$ & MS & 27 & $347 \times 11.5$ & $125 \times 22$ & HTS \\
\hline 12 & $647 \times 11.5$ & $125 \times 25$ & MS & 28 & $397 \times 25$ & & HTS \\
\hline 13 & $350 \times 25.4$ & & MS & 29 & $300 \times 35$ & & MS \\
\hline 14 & $647 \times 12.7$ & $150 \times 25$ & MS & 30 & $230 \times 12.7$ & & MS \\
\hline 15 & $697 \times 12.7$ & $150 \times 25$ & MS & 31 & $230 \times 12.7$ & & HTS \\
\hline 16 & $747 \times 12.7$ & $150 \times 25$ & MS & 32 & $397 \times 11.5$ & $100 \times 25$ & HTS \\
\hline
\end{tabular}

Table 3 Comparison between calculated ultimate moment and estimated moment at failure

\begin{tabular}{|c|c|c|c|c|c|c|c|c|}
\hline \multirow{2}{*}{ Method } & \multicolumn{9}{|c|}{ Ultimate Moment (MN.m) } \\
\cline { 2 - 10 } & \multicolumn{2}{|c|}{ Initial } & \multicolumn{2}{|c|}{$\%$} & \multicolumn{2}{c|}{ Est. corrosion } & \multicolumn{3}{c|}{$\%$} \\
\cline { 2 - 10 } & Hog & Sag & Hog & Sag & Hog & Sag & Hog & Sag \\
\hline Failure & 17940 & - & 92.8 & - & 17940 & - & 97.2 & - \\
\hline Yield moment & \multicolumn{2}{|c|}{19332} & \multicolumn{2}{|c|}{100.0} & \multicolumn{2}{|c|}{18457} & \multicolumn{2}{|c|}{100.0} \\
\hline Plastic moment & 22618 & \multicolumn{2}{|c|}{117.0} & 21533 & \multicolumn{2}{|c|}{116.7} \\
\hline Rutherford & 18979 & 15450 & 98.2 & 79.9 & 17860 & 14420 & 96.8 & 78.1 \\
\hline MARC-FEM & 20630 & - & 106.7 & - & 18520 & - & 100.3 & - \\
\hline Present & 19164 & 16392 & 99.1 & 84.8 & 17885 & 15232 & 96.9 & 82.5 \\
\hline
\end{tabular}

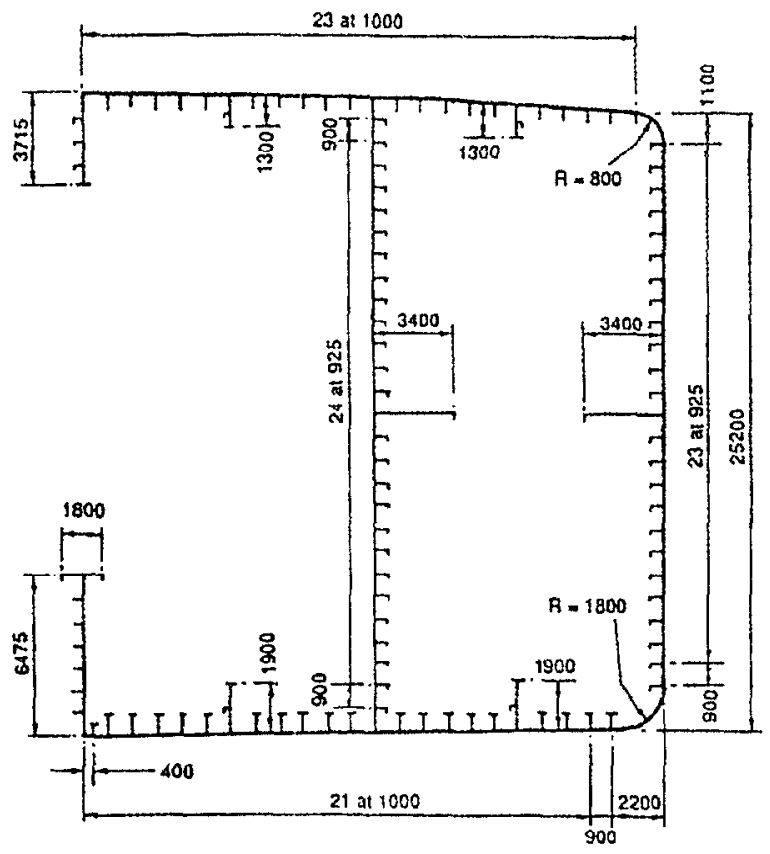

Fig. 9 Geometry and dimensions of Energy Concentration

buckling. The influence of the shedding pattern after buckling of the stiffened plates was tested and it was concluded that a large difference may be found in the ultimate moment using different shedding patterns.

The analysis of Energy Concentration is very interesting in the validation of these types of analyses because it shows how these simplified methods may predict and identify where the design of the ship may be improved in order to obtain better structural cross sections.

In this particular case, the ship had very slender panels in the deck and as a consequence they promoted the tripping of the stiffeners at an early stage of the loading followed by the premature collapse of the section at a low curvature, as shown in Fig. 11, and a very quick change in the position of the neutral axis.

It may be confirmed that there is no problem in the design of the bottom structure with respect to buckling and tripping, because the hogging ultimate moment is between the elastically derived yield bending moment and the plastic one.

\section{Study of effects of different phenomena on ultimate strength}

\section{Effect of corrosion}

During their lives, ships are subjected to the action of a corrosive environment. The external structures at the waterline and upper deck, as well as internal tank structures, are the areas most exposed to corrosion. The magnitude of the growing corrosion may be controlled by several techniques but, unless plates are replaced, a steady reduction of thickness takes place. Classification society rules normally include an allowance for corrosion, between a range of 0.5 and $3 \mathrm{~mm}$ depending on the position of the plate element, the nature of the tank and surrounding tanks, and the type of protection.

The present approach only considers a difference of corrosion rating between webs and flanges/plating, the former having double the corrosion rate of the latter. Each level of corrosion reduced the thickness of the plating and flanges by $0.5 \mathrm{~mm}$ and the web's thickness by $1 \mathrm{~mm}$. 


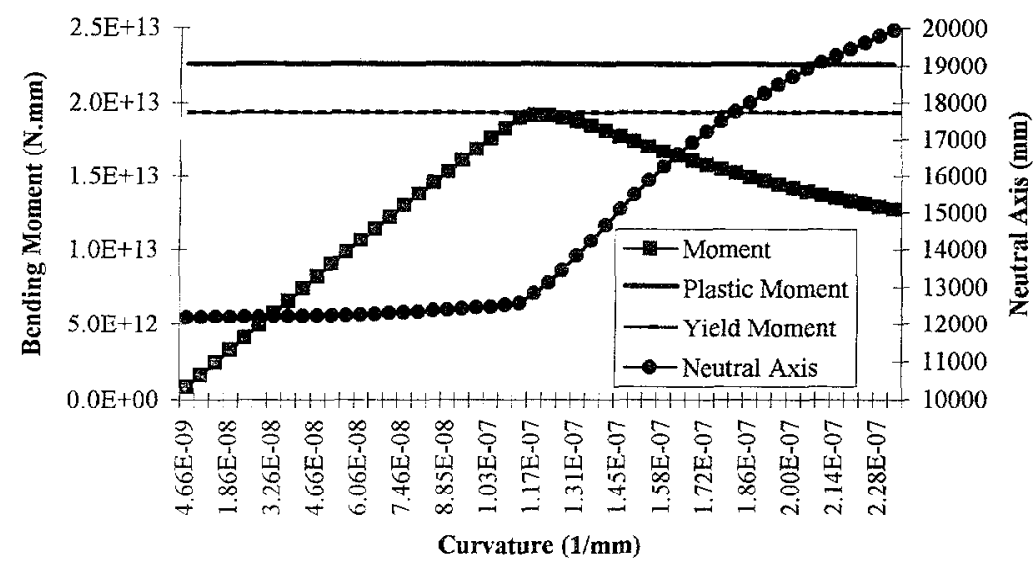

Fig. 10 Curve of bending moment and position of neutral axis in hogging ignoring residual stresses and corrosion

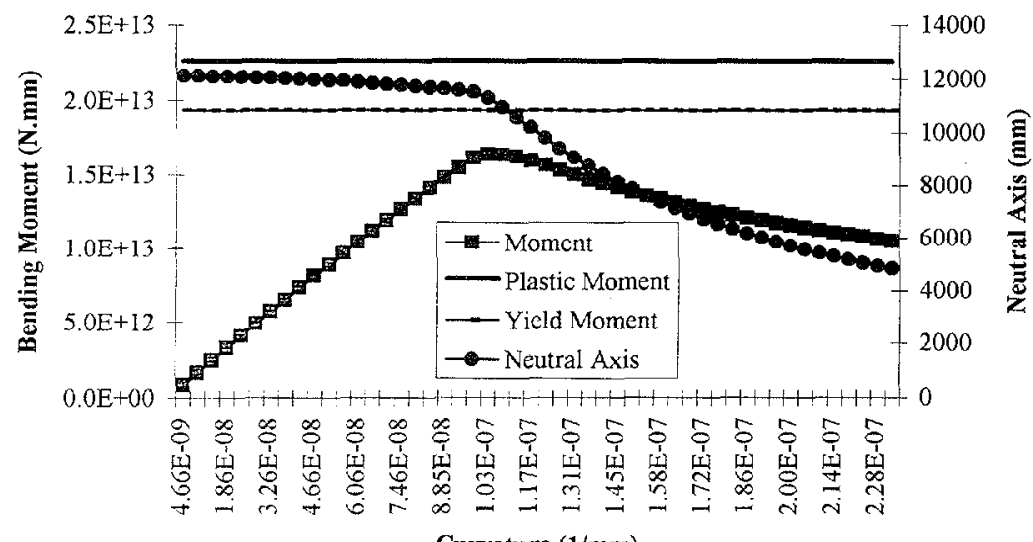

Fig. 11 Curve of bending moment and position of neutral axis in sagging ignoring residual stresses and corrosion

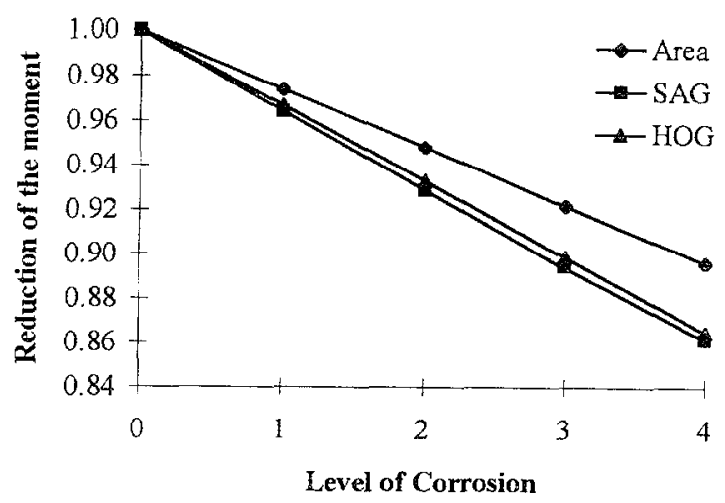

Fig. 12 Effect of corrosion on ultimate bending moment

Figure 12 shows the results for the total area, ultimate moment in hogging and sagging for each level of corrosion, comparing them with the values for the "new" ship in percentages. One may conclude that if the panels in compression are stocky (bottom panels in hogging), then $1 \%$ reduction on the total area in this ship promotes a reduction in ultimate moment of about $1.3 \%$. For sections with slender panels in compression, which is the case of the deck in sagging for this ship, the same reduction of $1 \%$ promates a reduction of $1.34 \%$ in the ultimate moment.

The figure shows the linear correlation of corrosion rate (or reduction of section area) and ultimate moment variation.

\section{Effect of residual stresses}

The presence of residual stresses in the plating usually leads to a reduction of the effective width of the plate elemonts with a consequent decrease in the column strength. The reduction on the ultimate strength of simply supported plates was already quantified by Faulkner (1975) and Guedes Soares $(1988,1992)$. However, if one is dealing with the load-end shortening curves, this information is necessary, but not sufficient. There is a need to know precisely the consequence of residual stresses in the pre- and post-buckling state of the plate. Thus, an approximate method (Gordo \& Guedes Soares 1993), similar to the one developed by Crisfield (1975), was implemented. The effect of residual stresses is incorporated directly in the material behavior, $\Phi_{c}\left(\bar{\epsilon}_{i}\right)$ of $(14)$, in such a way that the material behavior becomes

$$
\Phi_{e}\left(\bar{\epsilon}_{i}, \bar{\sigma}_{r}\right)=\max \left\{-1, \min \left(1, \bar{\epsilon}_{i}, \frac{\bar{\sigma}_{r} \cdot \bar{\epsilon}_{i}+1-\bar{\sigma}_{r}}{1+\bar{\sigma}_{r}}\right)\right\}
$$

where $\bar{\sigma}_{r}$ is the nundimensional residual stress, $\sigma_{n}=\sigma_{r} / \sigma_{o}=$ $2 \eta /(b / t-2 \eta)$.

Residual stresses are classified by levels, $\eta$, each of them corresponding to a tensile zone with a width of the level times the thickness of the plate. Using this method and assuming that $\eta$ depends on the welding process, then plates with different thicknesses will have different residual stresses $\bar{\sigma}_{r}$ to the same level of weld tension block $\eta$.

Figure 13 analyzes the effect of residual stresses on the 


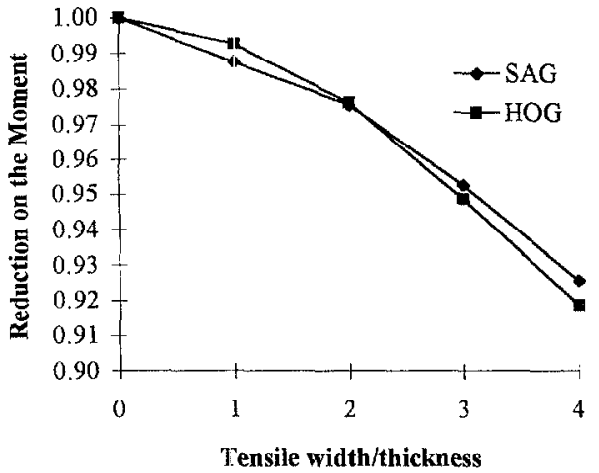

Fig. 13 Effect of residual stresses in sagging and hogging bending moment

ultimate bending moment for both sagging and hogging conditions.

The relation between the ultimate moment and $\eta$ may be considered nearly linear. In order to have a comparison, the level 3 of $\eta$ corresponds to $\bar{\sigma}_{r}=0.167$ in a plate having a width-thickness ratio of 40 . The deck and bottom plate elements of Energy Concentration have this typical value (1000 $\times 25 \mathrm{~mm}$ ) and a reduction of strength of $5.1 \%$ is predicted for hogging and $4.7 \%$ for sagging.

In sagging, the reduction on the strength is higher than in hogging at low $\eta$ and lower at high $\eta$. However, both curves are very much in accordance if one keeps in mind that the stiffened plates of the deck and bottom have very different column slendernesses.

As a main conclusion of this discussion, one may quantify the effect of residual stresses on ultimate bending moment for ships where tripping failure is avoided as being given approximately by

$$
M_{u r}=\left(1 \cdots 0.3 \cdot \bar{\sigma}_{r}\right) \cdot M_{u}
$$

where $M_{u r}$ and $M_{u}$ are the ultimate bending moment with and without residual stresses, respectively, and $\bar{\sigma}_{r}$ is the residual stresses of the panels in compression. This expression is based on compressed plate elements of $b / t=40$ (bottom panels in hogging) and consequently the dependence on this parameter has to be investigated.

\section{Analysis for combined loading}

Elastic analysis of the hull girder predicts that the behavior of a cross section of usual ships under combined vertical and horizontal bending can be described by an interaction equation which is an ellipse, where the elliptical distances are, respectively, $\sigma_{o} \cdot W_{v}$ and $\sigma_{o} \cdot W_{k} . W_{v}$ is the minimum of the section modulus of deck and bottom and $W_{h}$ is the section modulus of the side relative to the center line.

The hypotheses to be fulfilled in order to obtain this elliptical behavior are

- the distance from the deck and bottom to the neutral axis in the upright position must be constant and sides must be vertical; i.e., there is no rise of floor or camber, and

- the yield stress in compression must be the same as in tension.

As usual, ships do not fulfill the first requirement because there is always camber and bilge radii; the limit state curve in elastic theory is somewhat different from the ellipse due to the shift of the furthest point from the heeled neutral axis, $(x, y)$ in equation (18):

$$
M=\sigma_{\circ} \cdot \frac{I_{h} \cdot I_{v}}{x \cdot I_{v} \sin \theta-y \cdot I_{h} \cos \theta}
$$

The above equation is achieved from the limit stress state

$$
\sigma_{o}=\frac{M_{y} \cdot x}{I_{h}}-\frac{M_{x} \cdot y}{I_{v}}
$$

where the components of the bending moment in the principal directions are

$$
\begin{aligned}
& M_{x}=M \cdot \cos \theta \\
& M_{y}=M \cdot \sin \theta
\end{aligned}
$$

where $\theta$ is the angle between the moment vector and the horizontal axis, and the angle of the ncutral axis with the same axis, $\psi$, is given by

$$
\tan \psi \equiv \frac{y}{x}=\frac{I_{v}}{I_{h}} \cdot \tan \theta
$$

The total bending moment is related to the components by

$$
M^{2}=M_{x}^{2}+M_{y}^{2}
$$

Faulkner et al (1984) deduced from numerical studies a circular failure interaction equation for the HMS Cobra, which had rise of floor, camber, and some asymmetry in the cross section. This means that one may calculate the ultimate strength of a ship subjected to combined bending if the vertical and horizontal ultimate moments are known. However, some deviation to this circularity may be detected in particular ships and for particular ranges of angles of heel.

When the ship has camber, and successively increasing heeling angles are considered, the point $(x, y)$ to be used in equation (18) moves from the intersection of the centerline and deck towards the side corners, which corresponds to a smaller distance to the actual neutral axis than the initial one. As a result, the elastic moment is reduced slightly for small angles of heel when it might normally be expected to increase initially. This explanation is seen from the same behavior found from the elasto-plastic analysis, Fig. 14.

Several analyses were performed, covering all angles of heel from sagging lo hogging, as shown in Figs. 15 and 16, which give results when the neutral axis is kept at an angle of $160 \mathrm{deg}$ to the horizontal axis (20 deg from pure hogging). It is important to note from these figures the change in the direction of the moment vector and the dissimilar behavior of its horizontal and vertical components when curvature increases.

Initially the angle is about $139 \mathrm{deg}$ and this value may be computed with equation (22), which is valid when an elastic linear analysis is performed. When the curvature is increased the angle changes slightly towards the neutral axis, but this shift becomes very quick for curvatures near the buckling of the bottom and side plating, which corresponds to the maximum horizontal component of the bending moment. The moment vector moves its direction towards the neutral axis until the maximum of the vertical component, and even

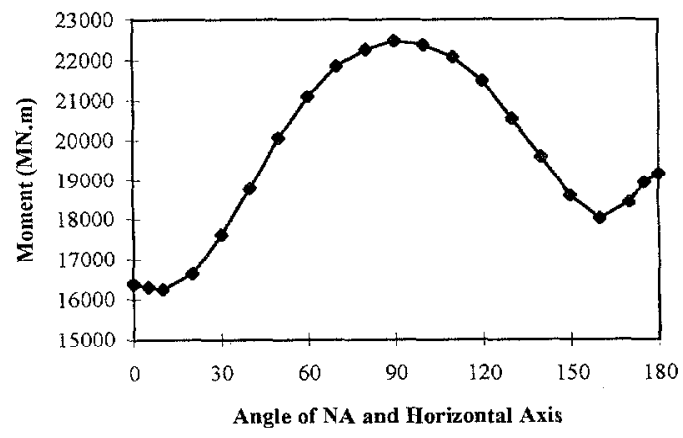

Fig. 14 Combined bending moment 


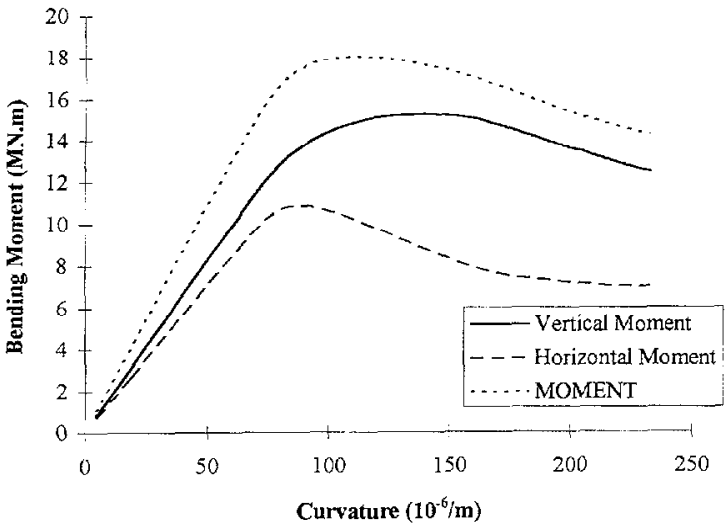

Fig. 15 Components of bending moment at $\psi=160 \mathrm{deg}$

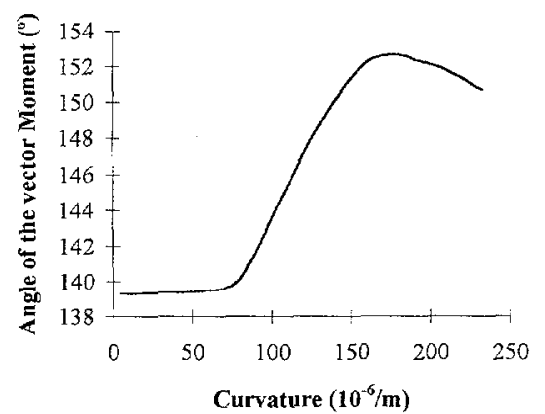

Fig. 16 Variation of angle of moment with curvature when neutral axis is located at $\psi=160 \mathrm{deg}$

further than that, because of the unloading in the horizontal moment.

This behavior is a consequence of the lack of proportionality between $M_{x}$ and $M_{y}$, which becomes important after the buckling of the first panels; i.e., because bottom and side panels have different column slenderness, the buckling and post-buckling behavior of the corresponding panels is different, thus the rate of change of the load-carrying capacity of vertical and horizontal moments changes with increasing curvature.

In the present case, at an angle of $160 \mathrm{deg}$, a collapse of the section is evident in $M_{y}$ at a curvature of $88 \times 10^{-6} / \mathrm{m}$, while $M_{x}$ has its maximum value at a curvature of $140 \times 10^{-6} / \mathrm{m}$. The explanation for this is that the side panels near the bilge collapse first and this collapse is more important for the horizontal than for the vertical modulus due to the greater reduction in effective inertia moment about the vertical than about the horizontal axis.

The impact of the buckling on the effective moment of inertia is a result of three main contributions

- reduction in the moment of inertia about the initial axis by an amount proportional to $\left(1-\Phi_{c}\right) \cdot x^{2}$ and $\left(1-\Phi_{c}\right) \cdot y^{2}$ for $y_{\xi}$ and $x_{s}$ axes, respectively, where $\Phi_{c}$ denotes the ultimate strength of the most compressed panels,

- reduction in effective section area, and

- the shift in the neutral axis.

The first is the most important contribution and because $y$ is normally greater than $x$, the impact on $I_{h}$ will be greater than on $I_{v}$.

Relative to the dependence of the ultimate moment with the angle of heel, the principal conclusions are

- the different results for hogging (180 deg) and sagging ( 0 deg) due to dissimilar types of collapse, as already commented upon, and

- the reduction of strength for small heeling angles, with minimums at 15 and $160 \mathrm{deg}$ for sagging and hogging.

\section{Efficiency of high-strength steel}

High-strength steel is one of the many choices at the disposal of the designer when longitudinal requirements are not fulfilled. This solution is extensively used in "long" ships such as VLCCs and ultra large crude carriers (ULCCs).

Apart from some problems with fatigue arising in crude carriers which use high tensile steel, or an eventual degradation of yield stress with aging, the objective of this subsection is to investigate the efficiency, from a structural point of view, of using HTS instead of mild steel.

The cross section of Energy Concentration was analyzed for three situations: using only mild steel of $235 \mathrm{MPa}$ yield, totally built of HTS (315 MPa), and "as built" using HTS in the bottom and deck and MS on side shell and bulkheads according to the general design practice for VLCCs. Table 4 shows the results of this analysis by comparing the method with the results expected using elastic analysis.

The main point to note is the dissimilar effects on the resistance when the panels in compression are stocky (hogging in this example) or slender (sagging).

From the elastic analysis a potential gain of $34 \%$ in longitudinal strength is expected when changing from MS to MS + HTS or HTS. In fact, only $27 \%$ is reached in the "best" condition of this particular ship, i.e., when the panels in compression are designed to avoid tripping and are sufficiently stocky.

The potential gain $\sigma_{H T S} / \sigma_{M S}$ is never achieved because the increase of yield stress increases the slendernesses of the plate and of the column element when the geometry is unchanged, which corresponds to a reduction on average effective stress supported by the panel.

\section{Conclusions}

The simplified procedure used in this work to predict the behavior of the hull girder under predominant longitudinal bending seems to be quite accurate when the results are compared with those obtained by different approaches and also in the case of a real ship failure.

On the other hand, if the preparation time of the model and the computational running time are considered and compared with methods using finite-element programs, then the developed procedure is far better and more useful for normal design practice. The simplified method takes about three minutes to run and calculate the whole moment-curvature curve in a 386 personal computer, while finite-element methods normally need a mainframe to run the model and several hours of CPU time. In relation to the preparation time of the model, finite-element methods may take more than a month to implement the model while the approximate method only requires about two days of work.

The method predicts a load-shedding pattern in the behavior of the stiffened plate after buckling. This prediction is based mainly on the assumed variation of the effective width of the associated plate and thus it requires confirmation by

Table 4 Effect of diffcrent steels on ultimate moment

\begin{tabular}{|c|c|c|c|c|c|c|}
\hline Steel & First yield & Sagging & $\%$ & Hogging & $\%$ & HOG/SAG (\%) \\
\hline MS-235 & 14422 & 13241 & 80.8 & 15114 & 78.9 & 114.1 \\
\hline MS-HTS & 19332 & 16392 & 100.0 & 19164 & 100.0 & 116.9 \\
\hline HTS-315 & 19332 & 16402 & 100.0 & 19190 & 100.1 & 117.0 \\
\hline
\end{tabular}


other methods-experiments or finite-element methods (Gordo \& Guedes Soares 1993). This confirmation is especially important for prediction of the ultimate strength of the ship cross section due to the different state of strain of each stiffened element, where some of them are already in the post-buckling region.

Another interesting feature is that the ultimate bending moment in the upright position is greater than the moment at small angles of heel, no matter if the ship is in hogging or sagging. Thus it is necessary to evaluate if this reduction should be included in the rules of the classification societies. In practice, if an elasto-plastic analysis of the hull girder is performed and the calculation of the minimum ultimate moment is required, then one should look for the minimum at an angle of heel of about $10 \mathrm{deg}$ or more. Alternatively, a reduction to the upright moment should be considered. However, the last solution seems to be worse than the first because the degree of the reduction depends on the compressive strength of the deck and side panels.

The use of HTS should be carefully evaluated by the designer because, from a structural point of view, the increase of the yield stress is not fully represented as an increase in the total strength; normally this increase is much lower than expected. If this small increase is judged, together with the higher price and the potential fatigue problems of the HTS, then the designer may conclude that HTS should be avoided in some cases.

Residual stresses and corrosion have a degrading effect on the ultimate moment. However, these effects occur at separate times in the ship's life; the residual stresses are present in the early stage of the ship life and the effect tends to reduce with the normal operating conditions, while the corrosion level tends to increase with time. So both effects should not be considered together for design purposes; i.e., if an allowance for corrosion is included in the design, then reduced allowance for the residual stresses should be considered.

Inclusion of a tripping formulation in the behavior of stiffened panels is seen to be very important in the particular case investigated here. The deck stiffeners, made of bars, don't have much flexural-torsional rigidity and the calculated tripping stress is lower than the flexural buckling stress of the stiffener with associated plate. This fact leads to a very high reduction of the ultimate bending moment in sagging compared with the moment in hogging, where the deck is in tension. Future work is desired on this subject and nowadays it is possible and relatively easy to compare the prediction of the tripping stress using approximate methods and the results of finite-element methods for stiffened panels.

The study of the impact of the residual stresses in plate elements developed here considered a particular case of stress distribution. However, a smooth transition between the tension and compression zones may easily be implemented by the modification of the equation which quantifies the change of the "material" behavior.

Future development of the approximate methods to predict the hull girder strength should include implementation of an explicit method to account for transverse strength of the hull and overall buckling of large panels. But, before this, it is very important to test extensively over the practical range of plate and column slenderness the derived load-end shortening curves of unstiffened and stiffened plates, investigating carefully the agreement in the post-buckling range.

\section{Acknowledgment}

The first author is grateful to JNICT, Junta Nacional de Investigação Cientifica e Tecnológica, for having provided a scholarship which supported his stay at the University of Glasgow, where part of the work was conducted.

\section{References}

ArAmCHAK, J. C. 1984 An approximate method for estimating the collapse of a ship's hull in preliminary design. In Proceedings, SNAME Ship Structure Symposium '84, 37-61.

AdaMchak, J. C. 1979 Design equations for tripping of stiffeners under inplane and lateral loads, DTNSRDC Report 79/064, Bethesda, Maryland.

BILLINGSLEY, D. W. 1980 Hull girder response to extreme bending moments. In Proceedings, 5th STAR Symposium, SNAME, 51-63.

CALDWELL, J, B. 1965 Ultimate longitudinal strength. Trans., RINA, $107,411-430$

CRISFIELD, M. A. 1975 Full range analysis of steel plates and stiffened plating under uniaxial compression. In Proceedings, Institution of Civil Engineers, 59, 595-624.

Dow, R. S., Hughl, R. C., Clarike, J. D., and Smith, C. S. 1981 Evaluation of ultimate ship hull strength. In Praceedings, Extreme Loads Response Symposium, SNAME, 133-147.

Faulkner, D. 1974 Compression strength of welded grillages, Ship Structural Design. Concepts, J. H. Evans, Ed.

FAULKNER, D. 1975 A review of effective plating for use in the analysis of stiffened plating in bending and compression, Journal of ShIP RESEARCH, 19, 1, 1-17.

FAULKNER, D. 1987 Toward a better understanding of compression induced tripping. Steel and Aluminum Structures, 3, R. Narayanan, Ed., Elsevier Applied Science, 159-175.

Faulkser, J. A., Clarke, J. D., Smith, C. S. and Faulkner, D. 1984 The loss of HMS Cobra-a reassessment. Trans., RINA, 125-151.

Gordo, J. M. ANd Guedes Soares, C. 1993 Approximate load shortening curves for stiffened plates under uniaxial compression. Integrity of Offshore Structures-5, D. Faulkner et al, Eds., EMAS, 189-211.

GoRDo, J. M. 1993 Longitudinal strength of ships, MSc. thesis, University of Glasgow, Scotland.

GUEDEs SOAREs, C. 1988 Design equation for the compressive strength of unstiffened plate elements with initial imperfections. Journat of Constructional Steel Research, 9, 287-310.

Guedes Soares, C. 1992 Design equation for ship plate under uniaxial compression. Journal of Constructional Steel Research, 12, 99-114.

Guedes Soares, C. AND Gordo, J. M. 1996 Compressive strength of unstiffened plate elements with initial imperfections. Journal of Constructional Steel Research, 16 (in press).

Lin, Y. T. 1985 Ship longitudinal strength. Ph.D. thesis, Dept. of NA\&OE, University of Glasgow, Scotland

Kutt, L. M., Piaszczyk, C. M., Chen, Y. K. And Lin, D. 1985 Evaluation of the longitudinal ultimate strength of various ship hull configurations. Trans., SNAME, 93, 33-53.

Rutherford, S. E. AND CatDwell, J. B. 1990 Ultimate longitudinal strength of ships: a case study. Trans., SNAME, 98, 441-471.

Sмrтr, C. 1977 Influence of local compression failure on ultimate longitudinal strength of a ship's hull. In Proceedings, International Symposium on Practical Design in Shipbuilding, PRADS 77, Tokyo, 73-79.

SMith, C. S. AND Dow, R. S. 1986 Ultimate Strength of a Ship's Hull under Biaxial Bending, ARE TR86204, ARE, Dunfermline, Scotland. 